\title{
Consideraciones teóricas para el análisis de las pequeñas y medianas empresas como fuente de generación de empleo
}

\author{
Pérez Prieto, María Elena*
}

\section{Resumen}

El objetivo del ensayo es analizar diferentes enfoques teóricos y empíricos sobre la veracidad de los argumentos que favorecen la idea de las Pymes como máximas productoras de empleo, para la cual se utilizó la metodología de la investigación documental con énfasis en bibliografía especializada. Se presentan argumentos a favor y en contra de la concepción, usualmente manejada, de cómo a través de esas empresas se dinamiza el campo laboral, y la creación de empleos estables y de calidad. Igualmente se abordan desde una perspectiva histórica crítica el surgimiento de las mismas, y la construcción de un mito económico a su favor. Los fundamentos teóricos se basan particularmente en los planteamientos de Harrinson, Los resultados del estudio indican estas juegan un papel muy importante en la estructura evolutiva del capitalismo mundial, pero no como líderes emergentes sino como seguidores de las prácticas de adaptación y rentabilidad de los grandes capitales, lo que sugiere modalidades distintas de observar el fenómeno de las Pymes como modelo de crecimiento económico y de generación de empleo.

Palabras clave: Pequeñas y medianas empresas, empleo, crecimiento económico, industria.

\section{Theoretical Considerations for Analyzing Small and Medium Enterprises as Sources for Generating Employment}

\section{Abstract \\ The objective of the essay is to analyze different theoretical and empirical approaches to argu- ments favoring the concept of small and medium enterprises as maximum producers of employment; \\ Recibido: 10-11-05. Aceptado: 25-01-07 \\ Licenciada en Administración. Mención Gerencia Industrial. Magíster en Gerencia de Recursos Humanos. Cursante del Doctorado en Ciencias Sociales. Mención: Gerencia de la Universidad del Zulia. Profesora Asociada, adscrita al Programa Administración de la Universidad Nacional Experimental Rafael María Baralt (UNERMB). E-mail: perezmarielena@cantv.net; perezmariele@hotmail.com.}


the methodology of documentary investigation with emphasis on specialized bibliography was used. Arguments for and against this concept are presented, commonly understood as how those companies make the labor field more dynamic and create stable, quality jobs. Likewise, their emergence and the construction of an economic myth in their favor are approached from a critical historical perspective. Theoretical foundations are based particularly on the statements of Harrinson. Results indicate that SaMEs play a very important role in the evolving structure of world-wide capitalism, not as emergent leaders but as followers of the adaptation and profitability practices of the great capitals, which suggests different modalities of observing the SaMEs phenomenon as a model for economic growth and generating employment.

Key words: Small and medium enterprises, employment, economic growth, industry.

\section{Introducción}

El estudio de las Pequeñas y Medianas Empresas (Pymes) como factor determinante para el crecimiento económico de un país, así como para la generación de empleo sustentable y de calidad, ha ocupado la atención de economistas, políticos y científicos del área, quienes consideran este sector empresarial como vital para fomentar los espacios productivos.

Al respecto Enright (1994), concluye que las Pymes juegan un papel significativo en las economías desarrolladas al generar una proporción sustancial de la producción y el empleo. Por otra parte Andriani, Biasca y Rodríguez (2005), afirman que las pequeñas y medianas empresas constituyen en cualquier país, el grupo predominante de empresas, en algunos casos superan el $99 \%$ de unidades económicas, contribuyen significativamente a la creación de empleo, y a la generación de riqueza. Con base a estas premisas, los gobiernos, particularmente en América Latina han diseñado y ejecutado un conjunto de medidas a fin de promover y fortalecer el desarrollo de este sector con la intensión de disminuir el índice de desempleo.
Tal como lo plantea Katz (2001: 176), al afirmar que, la promoción a la actividad de la Pymes no es una actividad reservada solo a países del sector industrial rezagado; al contrario las naciones más industrializadas mantienen una política industrial activa en este sector y sus gobiernos consideran como prioritario el apoyo a las Pymes, porque tales políticas favorecen no solo el desarrollo económico sino también el crecimiento del empleo. A pesar de los esfuerzos realizados por el Estado Venezolano, evidenciados en las consideraciones de orden jurídico, económico, fiscal, financiero y de promoción técnica, es relativamente positivo el impacto que estas medidas o políticas han tenido para mejorar las condiciones del empleo en el país.

Las ideas esbozadas permiten exponer como propósito de este ensayo analizar la pertinencia de lo enunciado en cuanto a la idea de las Pymes como máxima generadora de empleo. El estudio se concentra en la revisión teórica de los argumentos que favorecen tales afirmaciones, así como aquellos que los cuestionan con base a estudios científicos, en particular los relacionados con la cantidad y calidad del empleo generado por dichas empresas. Para tal efecto, se abor- 
da desde una dimensión histórica la génesis de estas creencias a los fines de justificar la evolución del pensamiento empresarial desde diversas perspectivas, así como la posición discursiva de los autores.

Cabe destacar que al hablar de las Pymes nos referimos al conglomerado de la pequeña y mediana empresa, según criterio cuantitativo adoptado por Venezuela son aquellas que agrupan hasta 100 trabajadores, considerando una subdivisión en dos sustratos, mediano inferior de 21 a 50 y mediano superior de 51 a 100 , la gran industria estará conformada entonces por más de 100 personas (Teran, 2002:22).

Este ensayo se abordó a través de la investigación documental, con la utilización de bibliografía y referencias especializadas en el tema.

\section{Desarrollo}

\subsection{Surgimiento de las Pymes}

Con respecto a diferentes perspectivas que implica el análisis del tema en discusión, en primer lugar puede citarse los argumentos emitido a favor de las Pymes como máxima generadoras de empleo estable y de calidad, los cuales de acuerdo a las consideraciones convencionales las grandes empresas y corporaciones en muchos aspectos se convirtieron en estructuras rígidas incapaces de competir en un mercado cada vez más exigente y especializado, donde las condiciones y exigencias de los clientes serian determinantes para definir las características de los productos. Por lo tanto, el peso de estas grandes empresas y sus modelos básicos de estandarización las llevaría inevitablemente al cierre, tal como lo anuncia Vermon (1997:103), al expresar que el proceso postindustrial exigía un nuevo modelo productivo que implicaba una disminución en la plataforma productiva y en las estructuras de costos presentes en la gran industrial.

Asimismo, Chandler (1977:103), resumía lo acontecido como. "La hecatombe del modelo industrializado y de producción en masa, como consecuencia del dinamismo propio del mercado emergente". Tal como se evidencia el aumento en el ingreso y el nivel de vida de los consumidores presionarian hacia la búsqueda de productos y servicios mejor adaptados a sus necesidades y orientados hacia la moda; los mercados mayoritarios quedaron saturados, la demanda sobre ropa y muebles se fragmentaron y, tanto la educación como la comunicación masiva facilitó y promovió la heterogeneidad creciente en los gustos de los consumidores.

Por lo tanto, en un mundo especialmente orientado hacia la moda, la agilidad en la identificación de nuevos deseos y en el lanzamiento de nuevos productos al mercado se convierte en la clave para vencer a la competencia. Todos estos planteamientos, según quienes los esgrimen, favorecen a las Pymes por su capacidad de adaptación, tecnología e información, así como la flexibilidad de sus estructuras productivas y de costos; aspectos que atentan contra la burocracia de las grandes empresas, centradas en lograr una producción estandarizada y masiva.

A la par de esas realidades se expande un proceso de opinión y de formulismo teórico, el cual tuvo su máxima expresión en los conceptos emitidos por la 
Fundación German Marshall a comienzos de 1985, en su teoría sobre el desarrollo y el crecimiento económico centrado en la pequeña y mediana empresa. Bajo el esquema del Estado desarrollador proyectaba el empuje económico así como la modernización industrial acelerada, aspecto que rompería los cordones de pobreza y desempleo; esto generó un concepto político proclive a la concepción de nuevos negocios, libre empresa y desarrollo.

A propósito de lo planteado, Carrison, citado por Harrison (1994:25) explica:

Es fácil entender porqué tanto la versión empresarial como la versión industrial de la teoría del crecimiento basado en pequeñas empresas se hizo tan popular en los años 80 especialmente entre los políticos. Un surgimiento de sentimientos localistas, aunado a la promesa de que cualquier ciudadano con iniciativa empresarial y mínimo de inversión podría competir y ser exitoso, atrajo el discurso político del momento, y con ello el apoyo y estímulo a esta propuesta.

Esos argumentos apuntan hacia la creencia, de que las Pymes son el producto de la evolución histórica necesaria del proceso postindustrial tras el agotamiento de los esquemas masivos y estandarizados de producción, y de las estructuras propuestas por el Fordismo, así como por la superioridad de las Pymes para generar desarrollo y bienestar.

En segundo lugar, resulta interesante contraponer a esa versión, lo expuesto por Harrison (1994:50), quien afirma que

...en lugar de disminuir, el poder económico concentrado está cambiando de forma, de alianza, de acuerdos tecnoló- gicos y financieros a corto y largo plazo entre las mismas empresas, con los gobiernos a todos los niveles y con legiones de pequeñas empresas (aunque no siempre es así) que actúan como proveedoras y subcontratistas.

Ha decir del autor, la producción estaba siendo cada vez más descentralizada, dado que los directivos intentaban aumentar su flexibilidad (es decir, sacar de si mismo lo mejor) frente a las inmensas barreras para entrar a los mercados y a la incertidumbre crónica propia de las condiciones políticas y de las diferentes demandas de los consumidores.

Igual argumento expone Terán (2002:40) al afirmar lo siguiente: para obtener utilidad y crecimiento en la nueva realidad del mercado, el capital utiliza el progreso técnico y el de comunicaciones para concentrarse solo en áreas específicas, que le garanticen el éxito del producto, el resto lo subcontrata como forma evasiva de la acción sindical.

En el mismo orden de ideas, Harrison (1994:52) presenta, cuanto menos, cinco explicaciones posibles a los supuestos cambios de la gran empresa a Pyme:

1. Desintegración vertical de las grandes empresas, con la finalidad de huir de las centrales sindicales, de los salarios elevados y de los climas antagónicos de negocio.

2. Clausuras concentradas entre las mayores empresas y unidades productivas.

3. Un desplazamiento secular desde la producción (con sus grandes infraestructuras, en general) hacia los servicios (con su pequeña estructura en general). 
4. La reducción estratégica de los grandes conglomerados como parte de una concentración hacia las actividades nucleares o intrínsecas.

5. Un auténtico crecimiento desproporcionado en la actividad de las pequeñas empresas.

En particular, la opinión de los autores apunta más hacia las primeras cuatro explicaciones, con mayor énfasis sobre reducción estratégica, con la intensión de atender las actividades nucleares y flexibilizar el resto de los procesos a través de la subcontratación.

Ese análisis sugiere que el proceso de crecimiento de las Pymes se debe básicamente a una estrategia de producción por parte de las grandes corporaciones, quienes ante las demandas crecientes y heterogéneas del mercado, así como la amplitud del mismo optaron por concentrar sus esfuerzos productivos en aquellas que le eran medulares y de diferenciación, al calificarlas de nucleares, transfiriendo el resto a los proveedores, que en su mayoría constituyen pequeñas y medianas empresas especializadas en áreas concretas, y que en ocasiones pertenecen a empleados calificados despedidos o auspiciados desde la misma empresa.

Esa estrategia obedece a fines concretos de desplazamiento y ahorro de costos. A lo planteado Harrinson (1994: 30) expresa:

Un creciente número de pequeñas empresas se convirtieron en elementos pertenecientes a la estrategia del núcleo para el aligeramiento de la producción de las grandes empresas esta es la causa de la disminución en el tamaño medio de las organizaciones y no cual- quier otro tipo de crecimiento espectacular en el conjunto de las pequeñas empresas.

\subsection{El mito de las Pymes como generadoras de empleo}

Algunos factores coincidieron para crear el mito sobre las propiedades económicas y de empleo de las Pymes. El cierre de obsoletos esqueletos industriales, aunado a una fuerte recesión económica a finales de los años 70 y comienzos de los ' 80 , así como la proliferación de pequeños y medianos negocios, propiciaron que las Pymes absorbieran buena parte de los trabajadores desplazados del gran sector empresarial e industrial, todo como producto de una nueva versión en el campo empresarial.

A decir de Harrison (1994:39), esta situación sucedió como consecuencia del fuerte declive en los balances de las mayores corporaciones durante los desastrosos ejercicios económicos comprendidos entre los años 1973 y 1983 , lo que propició fuertes reducciones en las plantillas de mandos intermedios y de mano de obra directa, junto a un continuado y permanente cierre de las factorías que habían quedado obsoletas y no a un crecimiento desmesurado, propiamente dicho, en el número de pequeñas empresas.

A esa situación se suma la tendencia casi generalizada de la mayoría de los intelectuales y economistas de la época y aún en la actualidad al esgrimir afirmaciones y recomendaciones sobre la supremacía de las Pymes como reproductoras de empleo, tal como lo expresa Vera (2001:34), al señalar la importancia de las 
Pymes, destaca: "con relación a la pequeña y mediana empresa se coincide en que es un elemento crucial para el desarrollo de la competitividad de un país y fortalecimiento de la economía". Por otra parte, Vergara (2001:25) concluye que: "Una manera de combatir el desempleo era a través de la creación de pequeños y medianos empresarios capacitados por el Estado".

Semejante apreciación tiene la Organización Internacional del Trabajo (OIT:1998), al afirmar:

tomando nota de que las pequeñas y medianas empresas, como factor esencial del crecimiento y del desarrollo económico proveen cada vez más la mayoría de los puestos de trabajo creados a escala mundial y pueden ayudar a crear un entorno propicio para la innovación y el espíritu empresarial".

Una de las opiniones más influyentes para la época en cuestión, y al considerar lo expuesto por Harrison (1994:53), resultó ser las estadísticas presentadas por David Birch, investigador asociado a la Universidad de Harvard y sus colaboradores en el año 1979, sobre el fenómeno de las Pymes como creadoras de nuevos empleos basados en la fuerza aglutinante de la pequeña y dinámica empresa. Es este mismo entorno, el que ha dado lugar a conceptos de políticas públicas como pueden ser los polos de desarrollo, la incubadora industrial, sustitución de importaciones, la desregularización gubernamental, los tratamientos impositivos preferentes para los fondos de riesgo y los parques tecnológicos, todos unidos con el claro interés de promocionar el crecimiento de pequeñas empresas, los cuales han sido aplicado en América Latina y no han tenido el éxito esperado si se revisan datos estadísticos de crecimiento económico, empleo y desarrollo.

Según el mencionado autor, esos planteamientos han encontrado eco privilegiado en los medios de comunicación norteamericanos y europeos, generalizando los resultados al resto de los países del tercer mundo. En síntesis, este autor expresa que, Birch fue el primer analista político que presentó y asoció los resultados estadísticos sobre las solicitudes de crédito de la inmensa mayoría de empresas norteamericanas reunidas por Dum \& Badstreet Corporation (D\&B) a finales de los años 70 . El esfuerzo de Birch, incluía la tabulación sobre los puestos de trabajo de las empresas facilitadas por D\&B, clasificándolas en nacimiento, muerte, crecimiento y declive de estos establecimientos en períodos de tres años. Así mismo, construyó árboles genealógicos que unían establecimientos individuales.

Tras algunos hallazgos, Birch con una metodología poco clara a decir del autor pretendió haber demostrado que las empresas, fábricas, tiendas y oficinas pequeñas eran las responsables de la inmensa mayoría de los nuevos empleos creados, igualmente llegó a la conclusión de que se podría atribuir un $88 \%$ de la creación neta de empleo en este periodo a las actividades de empresas con menos de veinte empleados y la conclusión más espectacular y de mayor influencia era que parecía que la prolífica tasa de nacimiento de nuevos negocios en todos los sectores y regiones de los Estado Unidos era el responsable de la mayoría de los nuevos empleos generados. Estas conclusiones fueron inmediatamente asumi- 
das, adornadas, celebradas y publicadas a una audiencia más amplia por el popular escritor Gilder, en Europa y comentado como válida y absoluta en el resto del tercer mundo.

Lo relatado por Harrinson, muestra cuál pudiera ser la génesis del mito de las Pymes como máximas generadoras de empleo y de empuje económico, si se considera que la mayoría de las iniciativas económicas tomadas en los países del tercer mundo provienen de recetas 0 modelos económicos pensados desde el mundo anglosajón y desde sus perspectivas históricas propias.

Harrison (1994:55) y un grupo de investigadores, cuestionan los aspectos metodológicos y de contenido del informe de Birch. Al respecto manifiesta:

Existe por descontado, serias limitaciones técnicas inherentes a los datos de D\&B (y que siendo sinceros para con la empresa nunca pretendieron ser más que relacionar el nacimiento y cierre de empresas, como consecuencias de las peticiones de crédito efectuado por las empresas). La tendencia al secretismo por parte de Birch, en lo relativo a los métodos de análisis y sus constantes cambios de posiciones ante las interrogantes no ha ayudado en absoluto a saber cuales son los números correctos. Así mismo, genera dudas con respecto a la relación de las variables y la manipulación arbitraria que hace a las mismas.

\subsection{Discusión sobre la veracidad del mito}

Estudio reciente sobre la veracidad de las Pymes como generadoras de em- pleo y de crecimiento económico sostenido, revelan una realidad distinta a lo planteado por Birch. En particular presentamos uno de los más relevantes estudios sobre la generación de puestos de trabajo en los principales países industrializados, auspiciados por el Instituto Internacional de Estudios sobre el Trabajo (IILS, sus siglas en inglés). Estas referencias tomadas de Harrison (1994:57), aclaran el panorama al respecto. Se utilizaron los informes de cada país con la finalidad de reunir las tendencias estadísticas básicas comparadas en las distribuciones de los puestos de trabajo en empresas y establecimientos.

En resumen, se tomó como referencia la cifra oficial de los 100 empleados como definición operacional de lo que se debe entender por pequeña y mediana empresa. Ante la evidencia de que la empresa promedio ha disminuido de tamaño en sus plantas productivas desde los años 70 , así como el número de centros de producción por empresa y el número de éstas legalmente constituidas ha crecido en la mayoría de los países. Los investigadores interpretaron tras el análisis de los datos, que la participación de los puestos de trabajo en la empresa y plantas que emplean menos de 100 trabajadores ha pasado de una distribución en forma $V$ en función del tiempo, disminuyendo después de la segunda guerra mundial, para crecer posteriormente en los años 80.

Ante los hallazgos mencionados, los expertos optaron por explicar el fenómeno como producto de la especialización flexible; es decir, de un desplazamiento del proceso productivo de las grandes empresas hacia los sectores pe- 
queños y medianos empresariales, los cuales se especializaron en áreas determinadas; estos hechos demuestran que no ha sido el crecimiento de las Pymes, lo que dinamiza el empleo sino que atiende espacios operativos dejados por la industria en su proceso de adaptación al mercado.

En ese mismo orden de ideas y con la intención de consolidar las evidencias planteadas, se presentan los resultados de otro estudio realizado por Harrison y sus colaboradores, quienes, a partir de estadísticas empresariales publicadas en 1987 por U.S census of manufacturers en los Estados Unidos, se evidenció que la participación de las pequeñas empresas apenas ha cambiado desde, por lo menos, finales de los años 50; tal como lo reconoce Harrison (1994:78), la proporción de norteamericanos que trabajaban en empresas pequeñas y en establecimientos individuales apenas ha cambiado desde principios de los años 60 .

Es importante continuar con las investigaciones sobre el tema y citando nuevamente a Harrison (1994:57), quien ha servido como fuente argumentativa del presente artículo, al referir sobre la realidad del sector en otras latitudes, plantea:

Al analizar los datos referentes a la situación de Japón, lo acontecido en los años que mediaron entre el final de la segunda guerra mundial y finales de los años 60 y principios de los 70 , refleja un período de gran crecimiento de las grandes empresas, ilustrado en el largo y bastante pronunciado declive de la participación de las empresas con menos de 100 empleados en el número global de los puestos de trabajo existen- tes. Durante los años 70 se produjo cierta modificación a los anteriores planteamientos, pero entre 1980 y 1983 se volvió a la tendencia inicialmente apuntada.

Igualmente en América Latina las estadísticas resultan abrumadoras, sobre el fracaso de las Pymes, los datos tomados de Serrano (2004:45) indican que en promedio el $80 \%$ de las Pymes fracasan antes de los cinco años, y el $90 \%$ no llega a los diez años, de acuerdo a la misma fuente citando cifras de la Comisión Económica para América Latina y el Caribe CEPAL (2003) en los países subdesarrollados entre un 50 y un $75 \%$ dejan de existir durante los primeros tres años. Este informe continuo presentando los indicadores de países como Chile en el cual durante 1996 el 25\% de las Pymes desaparecieron en el primer año, un 17\% en el segundo año, el $13 \%$ en el tercer año y un $11 \%$ fracasó en el cuarto año. Parecida situación se presenta en México en el cual el $75 \%$ de las nuevas empresas del sector mediano o pequeño deben cerrar sus operaciones apenas dos años después de iniciadas. Continuando con otro informe más reciente (2006) del mismo organismo internacional la brecha que existe entre las grandes empresas y las PYMES sigue siendo amplia en América Latina y el Caribe. Las primeras se caracterizan por ser modernas y altamente competitivas, mientras en el grupo de las últimas, el retraso tecnológico aparece como el denominador común.

En general, la participación del las PYMES en las exportaciones y en los sectores más dinámicos de la economía es escasa -estas generalmente se concentran en sectores como textiles, pren- 
das de vestir, artículos de cuero, calzado, etc.-. Sólo algunas de las PYMES de la región, han sido capaces de insertarse de forma exitosa tanto en los mercados internos como los internacionales, contribuyendo efectivamente en la estructura productiva de la economía.

Es por eso que se hace fundamental, en momentos en que América Latina y el Caribe parecen estar jugándose todas sus cartas a la apertura comercial y al libre mercado, la creación de políticas de apoyo a las PYMES. Sin ese apoyo, será muy difícil que estas empresas logren sobrevivir en un mundo cada día más competitivo y que sigan contribuyendo a combatir uno de los mayores males de la región: el desempleo.

Lo anterior pareciera conducir a una constante global en la mayoría de las economías mundiales, lo cual refleja cierto antagonismo histórico, entre el mito de las Pymes como máxima generadora de empleo duradero y la realidad de este sector.

\subsection{La calidad y condiciones del empleo generado por las Pymes}

Este aspecto luce relevante al valorar la condición de las Pymes como generadoras de empleo más allá del aspecto cuantitativo, el cual como se ha observado es ciertamente cuestionado por algunos resultados concretos de investigaciones científicas.

Con fines didácticos y de discusión, se presentaron los argumentos de quienes favorecen las condiciones del empleo en estas empresas y quienes cuestionan esta versión. Para tal efecto, se tomarán como indicadores el salario y las condiciones laborales. Según la Organi- zación Internacional del Trabajo la OIT (1998:132), "las pequeñas empresas son hoy las más grandes contribuyentes a la creación de empleos, en cuanto a número y calidad, puesto que pueden proveer un trabajo decente a millones de trabajadores que laboran en malas condiciones y atrapados en la pobreza".

Asimismo, a las Pymes se les relaciona como el empleo para las clases desposeídas, por considerar a estas de bajo nivel educativo y de condición poco óptima para el trabajo, tal como lo expresa el ya mencionado organismo: "se reconoce que las Pymes ofrecen a las mujeres y otros grupos habitualmente desfavorecidos, la posibilidad de acceder en mejores condiciones a un empleo productivo, duradero y de calidad en particular reduce la brecha del trabajo informal y el empleo formal".

A decir de Vera (2001:76), "La relación de cercanía y familiaridad entre los dueños de los empleados de las Pymes genera condiciones de trabajo concertadas, propicias para la productividad". Estas razones apuntan a una situación ideal donde los intereses de ambos sectores parecen conciliarse gracias al contacto permanente y de comunicación abierta, cuestión que es colocado en duda por otros autores, tal como se verá más adelante. En concordancia con lo expuesto por Vera, Vergara (2001:274) afirma que, en general, "las Pymes cumplen con los lineamientos mínimos del trabajo en cuanto a sueldo, prestaciones sociales y ambiente de trabajo, por considerarse vulnerables a demandas y juicios por parte del Estado y de los articulares".

Por otra parte, se esgrimen argumentos, tales como la localización co- 
mercial de estas empresas, por lo general en sitios urbanos y céntricos, esto permite ahorrar en tiempo de viaje y dinero al trabajador, quien a diferencia de la gran industria debía transportarse a zonas específicas, en la periferia de la ciudad.

Igualmente, se plantea la posibilidad del reclamo inmediato, así como la estabilidad en cuanto a empleo por los costos de reposición de mano de obra que resulta muy elevada para el empresario, quien prefería mantener a un trabajador, aún cuando no lo considerase altamente efectivo, antes que asumir el despido injustificado y su correspondiente pago.

Resulta interesante escudriñar el fondo del asunto considerando; lo expuesto por Harrison (1994:55), al citar los resultados de las investigaciones realizadas por Brown, Hamilton y Medoff en el contexto norteamericano de los años 80 , al respecto expone:

"ellos y otros que han analizado la cuestión, llegaron, sin excepción, a la conclusión que después de contabilizar las diferencias entre industria $y$, en algunos casos, incluso entre empresas, en lo referente a disponibilidad de recursos humanos, a su formación, a sus condiciones laborales, a su edad y su relación con los sindicatos, los empleados de las grandes empresas disfrutan, por regla general, de salarios más altos, mejores beneficios sociales y mayor seguridad de empleo que sus equivalentes en las pequeñas empresas".

Esos hallazgos se repiten prácticamente en su totalidad en la mayoría de las economías desarrolladas.

Al seguir con el mencionado autor, quien reseña "el ILS de Ginebra, estable- ció una comparación muy meticulosa de las diferencias de salarios y los beneficios sociales entre Estados Unidos y Japón, en función de los tamaños de las empresas y de las plantas de fabricación. En ambos países los salarios promedios en los negocios más pequeños son menores que los negocios mayores. Con datos más actualizados y en relación a la realidad Venezolana, Páez (2004), plantea una diferencia significativa entre la calidad del empleo de las pequeñas y medianas empresas al expresar la diferencia en los beneficios sociales entre empresas grandes y pequeñas es aún mayor considerando el sector que más beneficios otorga dentro del conglomerado pyme en general como to es el sector industrial medio. El mismo autor expresa con preocupación que según el Observatorio de Empresas realizado por el Centro de Asesoría Técnica para la Producción Organizacional Ceatpro, (2007), en Venezuela estaban establecidas 480 mil empresas en general, ya para el 2004 se reportó el cierre de $6 \%$ de esa plantilla y de acuerdo al último estudio de 2006 aplicado a unos 3 mil establecimientos, ha desaparecido el $6 \%$ de la mediana empresa venezolana, además de reducido su tamaño, de 70 disminuyó a 30 el número de empleos que creaban estos establecimientos. De las 450 mil empresas que hay en la actualidad, según Páez, 99,02\% son Pyme que aportan el $70 \%$ del empleo formal del país. El $76 \%$ de la totalidad de las empresas tienen menos de 6 trabajadores, lo que significa que son consideradas microempresas, de ellas, $40 \%$ no tiene acceso a computadoras y los niveles de conexión a Internet de las que operan en las regiones del país con mayor desarrollo es 
de $25 \%$ y $10 \%$ las ubicadas en regiones de menos desarrollo en Venezuela.

Asimismo y citando fuentes de la OIT (2004:132), "el menor número de sindicalización se observa en las Pymes, dejando desprovistos a los trabajadores de los núcleos de reclamo". Con esta afirmación se manifiesta la preocupación por el hecho que los trabajadores de estas empresas no gozan de planes de jubilación, de paquetes contractuales propios o discutidos, así como de la higiene y seguridad propia del área de trabajo, en este orden de idea se expresa la información aportada por el Servicio Nacional Integral de Administración Aduanera y Tributaria SENIAT (2006) el sector mediano y pequeño tanto industrial como de servicio ha incumplido mayoritariamente su responsabilidad social con los aportes al seguro social obligatorio, y constituye el sector con mayor índice mora.

Insistentemente se presenta al trabajador Pyme como un recurso poco capacitado y de bajo adiestramiento, en particular por la no inversión de las empresas en este aspecto, cuestión que se utiliza para justificar los bajos sueldos y salarios. Según lo expresado por Andriani y Otros (2005), las personas se encuentran desmotivadas, no se dan las condiciones para que liberen su potencial y hagan contribuciones significativas, impulsando a la empresa a que se eleve constantemente su nivel de competitividad. Todas estas condiciones generan preocupación, ya que en ausencia de regulación por parte del Estado que permitan modificarlas, un programa económi$\mathrm{co}$, que tenga como objetivo un tratamiento especial para las pequeñas y medianas empresas, sin garantías de res- ponsabilidad social en cuanto a la contribución de empleos dignos y sustentables y entornos creativos donde se exprese la potencialidad de las personas y una gerencia efectiva que direccione los esfuerzos productivos hacia estándares de eficiencia, se podría estar impulsando un modelo que si bien es cierto resuelve buena parte de aspectos marginales del desempleo, no responde a un concepto de humanístico de la gerencia.

\section{Consideraciones finales}

Las evidencias empíricas demuestran las dificultades que presentan los gobiernos para estimular la creación de empleos seguros y sustentables. Pudiera reconocerse los esfuerzos políticos y económicos que en particular se han realizado en América Latina para compensar los altos niveles de desocupación y pobreza. Tras la búsqueda del desarrollo sustentable, y abrumados por sus propias realidades, se afianza en modelos y perspectivas orientadas por los países industrializados con base a sus aciertos y desaciertos.

Con base a esas condiciones, se ha venido impulsando un modelo de crecimiento económico basado en la promoción y apoyo de las Pymes como máximas generadoras de empleo. Esta visión queda fuertemente cuestionada desde el origen mismo de la creencia hasta su aplicación en la realidad, pues básicamente el gran sector industrial ha configurado una nueva presentación ante el mercado.

La idea expresa el hecho de que una incertidumbre creciente en la demanda global para nuevos productos y un mayor proceso de heterogeneidad en los 
gustos, confiere ventajas competitivas específicas para la pequeña empresa que pueden adaptarse rápidamente a los diferentes nichos del mercado y modificar, de forma flexible, su papel específico en las redes de producción determinadas por las grandes empresas.

En otras palabras, mientras la gran empresa y sus plantas productivas pueden haber reducido su importancia por el número de cierre de las mismas, quizás no hayan bajado necesariamente su contribución relativa en la renta nacional, en la producción, en los beneficios y en la generación de empleos de calidad. Esta representación de lo ocurrido pareciera concretizar el hecho de que estas organizaciones juegan un papel muy importante en la estructura evolutiva del capitalismo mundial, pero no como líderes emergentes sino como seguidores de las prácticas de adaptación y rentabilidad de los grandes capitales, lo que sugiere modalidades distintas de observar el fenómeno como modelos de crecimiento económico y de generación de empleo, en particular si se observan las cifras presentadas por observatorio Pymes de Venezuela (2005), el cual demuestra que el $57 \%$ de las empresas medianas y pequeñas fracasaron al año de haber iniciado operaciones, y el $80 \%$ tuvo los mismos resultado antes de cumplir los primeros cinco años, lo que demuestra la inestabilidad del empleo creado por este sector, y lo poco sustentable de la propuesta,

La utilidad de este sector estaría, especialmente en la ayuda que pueda prestar para la construcción de un entorno económico coherente $y$, tal vez, más estable en los sectores con bajos ingresos, de bajo poder adquisitivo y de inicia- tiva productiva en áreas específicas poco explotadas, en especial en el espacio comercial, así mismo esta modalidad se convierte en alternativa de creación para emprendedores de clase media y vía de para atender el problema del empleo informal.

Las Pymes deben ser observadas como entes productivos capaces de adaptarse y de evolucionar en el aspecto empresarial e industrial, pero sin olvidar el compromiso social que estas tienen en cuanto a la creación de espacios productivos dignos, seguros, confiables y bien remunerados para sus empleados. De lo contrario, el Estado deberá revisar sus prioridades en la formulación de políticas públicas para el sector empresarial e industrial medio, pues si bien es cierto que el mismo contribuye significativamente en la creación de empleo las condiciones de estos están por lo general por debajo del promedio exigido, incluso por ley en cuanto a beneficios sociales, por lo tanto el aporte debe orientarse cada vez más hacia el logro de beneficios colectivos, para mejorar la estructura del empleo nacional.

Asimismo, sin pretender menospreciar el aporte de las Pymes al complejo entramado económico, es necesario ensayar otras propuestas empresariales que potencien, no sólo el aspecto productivo y de servicios, sino también que orienten la acción laboral hacia un concepto más humano, en el cual se pondere la relación patrono-trabajador como una relación de correspondencia, igualdad e interacción, en la cual los intereses de ambos sectores redundan en función de los intereses colectivos y del país.

Para tal efecto se debe concentrar en los aspectos de gestión, ele- 
vando el modelo a nivel de competitividad, tal como lo plantea Andriani y Otros (2005) El sistema de gestión de las Pymes debe tener una direccionalidad clara con aspectos tales como la visión, misión, valores, objetivos, políticas, medidas de calidad entre otros, así como garantías de retorno de capital, todo esto aunado ha procesos continuos de capacitación y desarrollo del personal pudieran afianzar las posibilidades de éxito y con ello las condiciones del empleo ofertado por este sector.

\section{Referencias Bibliográficas}

Adriani, C.; Biasca, R.; y Rodríguez, M. (2005). Un nuevo sistema de gestión para lograr Pymes de clase mundial. México. Grupo Editorial Norma.

Birch L., David (1987). "The Job Generation Process". mimeo, MIT Program onNeighbourhood and Regional Change.

Centro de Asesoría Técnica para la Producción Organizacional CEATPRO (2007). Página Web del Centro.

Comisión Económica para América Latina y el Caribe CEPAL (2003). Informe económico y financiero del sector pequeño y mediano.

Comisión Económica para América Latina y el Caribe CEPAL (2006). Informe sobre indicadores de gestión.

Chandler, A. (1977). The visible Hand Cambridge. Massachusetts: s/e.

Enright, M. (1994). El reto de la competitividad. Venezuela: Ediciones IESA.

German Marshall Fund (1985).

Harrison, B. (1994). La empresa que viene. Barcelona: Ediciones Paidos, SAICI.
Indestry, M. and T. (1975). Londres: Macrrillan. Katz, J. (2001). Una visión global de las experiencias acumuladas y lecciones aprendidas sobre política industrial en América Latina. Buenos Aires. SELA.

Organización Internacional de Trabajo (1998). 132 Conferencias del organismo. Ginebra Informe anual.

Organización Internacional del Trabajo OIT (2004). 145 Conferencia del organismo. Ginebra.

Páez, T. (2004). Estudio de la pequeña y mediana empresa en Venezuela y las nuevas tecnologías de información y la comunicación. Caracas. Editorial Norma.

Serrano, L. (2004). Relaciones Laborales y de empresa en América Latina. Venezuela: Editores Vadell Hermanos.

Servicio Nacional Integral de Administración Aduanera y Tributaria SENIAT (2006). Página Web del organismo.

Terán, A. (2002). "La sustitución de importaciones en Venezuela y el modelo rentista industrial". Revista Venezolana de Ciencias Sociales. Vol. 15, No. 8 UNERMB, Postgrado. pp. 18-43. Cabimas.

Vergara, M. (2001). Microempresas y Microempresarios: una visión total. Venezuela: Editores Vadell Hermanos.

Vera, M. (2001). "Gestión Financiera de la PYMI de la ciudad de Maracaibo". Revista de Ciencias Sociales. Vol. 7. No. 1. Universidad del Zulia. Facultad de Ciencias Económicas y Sociales. Maracaibo. pp. 65-89.

Vermon, G. (1984). The spirit of enterprise. Nueva York: Basic. Books. 